\title{
Directed self-assembly of carbon nanotubes into microspheres
}

\author{
Mihye Choi ${ }^{1}$ and Youngnam Cho ${ }^{1,2 *}$ \\ ${ }^{1}$ Biomarker Branch, National Cancer Center, Goyang, South Korea \\ ${ }^{2}$ Cancer Biomedical Science, Graduate School of Cancer Science and Policy, Goyang, South Korea
}

\begin{abstract}
Carbon nanotube microspheres (CNMs) were prepared using the inherent self-organizing characteristics of the nanotubes that primarily rely on electrostatic interaction, not the assistance of any polymers or surfactants. They permit significant linkage with a variety of chemical species such as biomolecules, metal nanoparticles, and fluorescent dyes. The cationic species of guest molecules facilitate individual nanotubes to coalesce and merge together into new colloidal structures. These novel CNMs create new technical opportunities for a wide range of applications. In particular, the capability of conjugating with a broad number of chemical species would be a fundamental basis for development of bio-electronics and biomedical applications.
\end{abstract}

\section{Introduction}

There has been great interest in developing novel strategies regarding fabrication, characterization, and possible applications of carbon nanotubes (CNTs) [1-3]. Recent advancements extend their applications to areas as diverse as electronic devices, environmental fields, and the biotechnology industry given their unique electrical, mechanical, and thermal characteristics as well as high surface area [4-6]. However, the difficulties associated with poor solubility and self-aggregation of pristine nanotubes present limitations in further processing and often restrict the practical use of CNT, especially for micro / nanoscale devices. A number of studies have focused on manipulating the self-assembly nature of nanotubes. The most common techniques involve the use of dispersants such as surfactants, solvents, polymers, or biomolecules with the hope of facilitating stable dispersion through covalent or non-covalent functionalization of CNTs [7-9]. Another approach involves processing highly aligned nanotubes in the form of bundles with morphological diversity to fabricate mechanically strong and conductive composites such as fibers, pellets, sheets, and yarns [10-15]. Such densely packed and well-aligned microscopic forms of CNTs possess large reactive areas and are durable, while simultaneously retaining fundamental properties of individual nanotubes. A recent advancement was the development of CNT hybrid materials with planar or spherical geometries [16-18]. In fact, the addition of the nanotubes into polymers usually alters the physicochemical properties of the films, endowing a remarkable combination of conductivity and mechanical robustness [19,20]. Several groups have pursued CNT-based capsules by adopting layerby-layer (LBL) deposition techniques [21-24]. CNT capsules can be produced through the self-assembly of oppositely charged polymers and CNTs in a water-in-oil emulsion system or with the use of colloidal templates. The size and flexible functionalities of the microcapsules rely on the diameter of the template particles and chemical species used. Notably, hollow microcapsules with large interior spaces are useful for the efficient loading and release of a variety of guest molecules [21,25]. Despite these advancements, very significant challenges remain, including complications in fabrication and the collapse of the spherical architecture after the removal of core templates. In previous study, our group has demonstrated the formation of mechanically robust, stable, surfactant-mediated carbon nanotube microspheres using a series of surfactants with cationic, anionic, and non-ionic charges [26]. In parallel with these advances, we provide a simple approach for the large-scale construction of spherical, water-stabilized CNT microspheres (CNMs) with a mean diameter of $\sim 17 \mu \mathrm{m}$ as illustrated in Figure 1a.

Additionally, we report the formation of CNM complexes incorporating various chemical species including biomolecules and metallic nanoparticles, Figure $1 \mathrm{~b}$. Our novel CNMs were obtained in a 'green' water-based process since surfactants, polymers, harsh chemicals, or solid templates were not used during fabrication.

\section{Experimental}

\section{The fabrication of CNMs}

The fabrication of water-soluble CNMs was prepared as follows. Four different types of multi-walled carbon nanotubes (MWCNT; Nanolab Inc, MA) were used: (1) pristine; (2) amine-modified nanotubes (diameters of $30 \mathrm{~nm}$ and lengths of $1 \sim 5 \mu \mathrm{m}$ ); (3) carboxyl $(\mathrm{COOH})$ groups-decorated (diameter of $30 \mathrm{~nm}$ and lengths in the range of $1 \sim 5 \mu \mathrm{m}$ ); and (4) short COOH-decorated nanotubes (diameters of $30 \mathrm{~nm}$ and lengths in the range of $500 \mathrm{~nm} \sim 1 \mu \mathrm{m}$ ). According to the company, carboxylated nanotubes have $2-7 \mathrm{wt} \% \mathrm{COOH}$ by titration after functionalization and metal catalyst impurities would be around $3-4 \%$. Pristine and functionalized nanotube dispersion with typically

*Correspondence to: Cho Y, Biomarker Branch, National Cancer Center, Goyang, Gyeonggi-do 410-769, South Korea, E-mail: yncho@ncc.re.kr

Key words: carbon nanotube, microspheres, SEM, biomolecules incorporation, fluorescence-loaded spheres

Received: May 25, 2018; Accepted: June 06, 2018; Published: June 08, 2018 


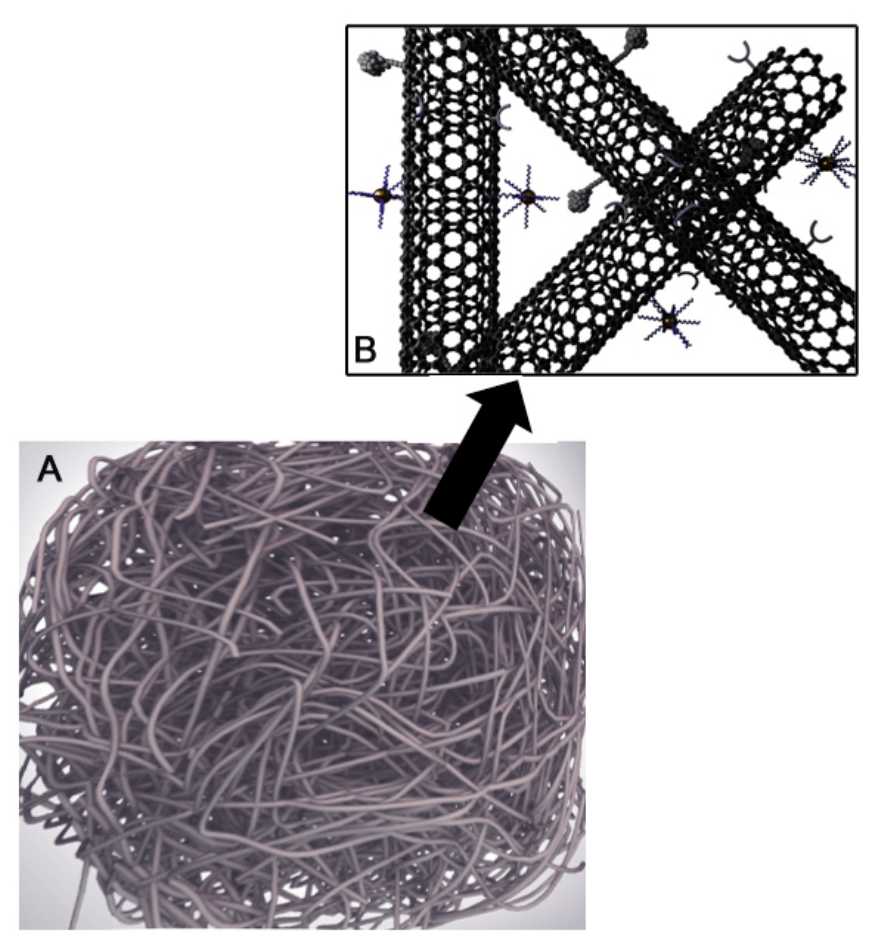

Figure 1. Illustration showing the preparation of CNMs and CNM-biomolecules complexes. (a) exhibits the fabricated CNM in the center. (b) shows prospective applications depicting a variety of biomolecules loaded onto nanotubes.

$0.1 \mathrm{ml} / \mathrm{mL}$ were prepared by adding MWCNTs into milli-Q water and then sonicated using high power ultrasonics for $5 \mathrm{~min}$. The dispersion was subsequently treated in an ultrasonic bath for $1 \mathrm{hr}$ and then subjected to high-power sonication again for $5 \mathrm{~min}$. After this, the $\mathrm{pH}$ of samples was adjusted to 2, 7, or 11 by adding $0.1 \mathrm{M} \mathrm{HCl}$ or $\mathrm{NaOH}$ to allow unstable bundles to be modulated into spherical morphology. The final samples were further purified with DI water via centrifugation and redispersion cycles. After repeating the process three to five times, resulting materials were dried in a vacuum oven at $40{ }^{\circ} \mathrm{C}$. Through a series of these steps, only carboxyl $(\mathrm{COOH})$ groups-decorated (diameter of $30 \mathrm{~nm}$ and lengths in the range of $1 \sim 5 \mu \mathrm{m}$ ) nanotubes have allowed obtaining stable spherical agglomerates without any damage to their resulting structure during the washing and drying treatments that is further demonstrate by SEM. Continually, the conjugation of poly-L-lysine (PLL; Mw=70,000 150,000; Aldrich), bovine serum albumin(BSA), or amine-functionalized gold nanoparticles $(\sim 100 \mathrm{~nm}$ $\mathrm{Au}-\mathrm{NH}_{2}$; Nanopartz, Inc) within CNMs was achieved by mixing the aqueous solution containing $\mathrm{COOH}$-decorated nanotubes (diameter of $30 \mathrm{~nm}$ and lengths in the range of $1 \sim 5 \mu \mathrm{m}$ ) of $0.1 \mathrm{mg} / \mathrm{mL}$ with $100 \mu \mathrm{M}$ $\mathrm{PLL}, 100 \mu \mathrm{M} \mathrm{BSA}$, or $50 \mu \mathrm{M} \mathrm{Au}-\mathrm{NH}_{2}$ at various $\mathrm{pH}$ to allow spontaneous chemical adsorption. Unbound materials were thoroughly removed by centrifugation. The fluorescent dye-labeled CNMs were obtained by mixing $1 \mathrm{mM}$ FITC (Aldrich), $1 \mathrm{mM}$ rhodaimne 123 (Aldrich), 100 $\mu \mathrm{M}$ of FITC-PLL (Nanocs), or $100 \mu \mathrm{M}$ of Rhodamine-BSA (Nanocs) with $0.1 \mathrm{mg} / \mathrm{mL}$ carboxylic acid-decorated nanotubes solution at various $\mathrm{pH}$. The solution was then centrifuged and reconstituted until no fluorescence was detected in the supernatant. Finally, fluorescent $\mathrm{CNMs}$ were air-dried at room temperature.

\section{The characterization of CNMs}

Sample of CNMs were dried onto glass coverslips, and sputtercoated with AuPd (Hummer II sputter-coater, Anatech USA, Union
City, CA) prior to imaging with a FEI NOVA nanoSEM (FE Company, Hillsboro OR). Imaging was done with an Everhart-Thornley (ET) detector or Through-the-Lens high resolution detector (TLD) using $5 \mathrm{kV}$ accelerating voltage. The focused ion beam (FIB) ablation, used to analyze the inner structure of CNMs, was done with an FEI Quanta 3D FEG dual beam FESEM. Pt deposition protected the CNM surface during FIB milling. Milling parameters were $30 \mathrm{kV}, 10 \mathrm{~mm} \mathrm{WD}$ and 520 tilt. Images were captured using the ET detector at $5 \mathrm{kV}$ accelerating voltage.

\section{Results and Discussion}

The intertube interactions as a consequence of van der Waals attraction and hydrophobic bonds cause them self-aggregate in both organic and an aqueous medium. Moreover, electrostatic interaction through excess charges present on nanotube surfaces induces an additional aggregation which might involve their processing in stable spherical assembly with significant structural integrity. Indeed, our results suggest that electrostatic forces seem to be critical for determining controllable colloidal shapes as microsphere stabilization depends on the transition of the carboxyl group $(\mathrm{COOH})$ present on the nanotubes into ionized carboxylic acid species (COO-). Notably, a subtle difference in the density of the carboxyl group on the nanotubes has caused a significant impact on the geometric structure. Greater attractive interaction was observed at high $\mathrm{pH}$, where increased deprotonation of $-\mathrm{COOH}$ groups preferentially react with adjacent positive ions derived from the basic aqueous solution. Scanning electron microscopy (SEM) was used to monitor the CNM formation. High magnification images show CNMs with tightly packed but relatively smooth exterior surfaces (Figures $2 \mathrm{a}$ and $2 \mathrm{~b}$ ). The structures retain the rigid form while the aggregates of CNTs making up the structure still provide for large surface area. The presence of reactive surfaces provides several technical or scientific advantages given that: i) increasing the active surface area would be beneficial for supporting a variety of molecular species and ii) a randomly-connected nanotube network permits the free flow of gas or liquids through the CNMs.

In fact, tailoring a surface-to-volume ratio within the microspheres would be essential for extending the application of CNMs through the incorporation of reactive species. Thus, it is also necessary to understand the inner structure of CNMs. Focused ion beam (FIB) milling was used to ablate a portion of single CNM so as to observe transverse CNM in cross-section. This technique provided a unique view inside the spheres revealing the distribution of the nanotubes, the presence of defects that might exist, and the impurities that might be created during the nucleation and growth of the microspheres. As shown in Figures $2 \mathrm{~d}$ and $2 \mathrm{e}$, the interior analysis of microspheres confirmed the absence of crystallized chemical impurities and apparent structural defects. However, these colloidal aggregates tend to collapse, flatten, or become stretched into the substrate surface when $\mathrm{pH}$ is decreased [Figure S1] in Supporting Online Materials (SOM)]. Indeed, protonation / deprotonation process of negatively charged carboxylic acid species is essentially responsible for the dissociation/assembly of CNMs.

The current fabrication method for producing the CNM results in a size distribution. There are a number of factors that are crucial for governing the fabrication process, including the concentration, length, and surface charge of the nanotubes as well as reaction time and temperature. The length of the nanotubes appears to be more relevant in determining the final diameter and morphology of the microspheres than the concentration and experimental conditions. 

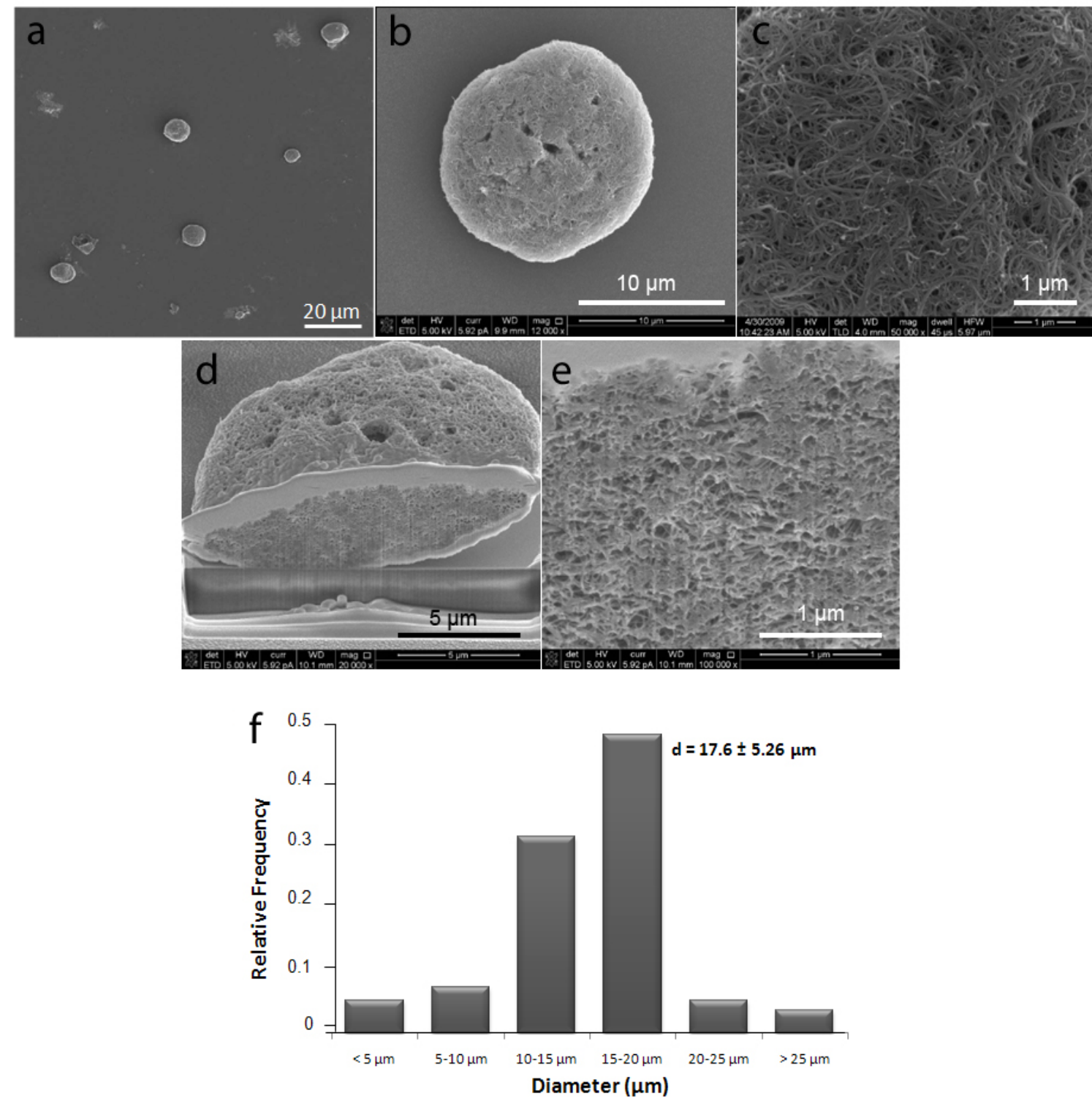

Figure 2. (a) (b) SEM images of self-supporting CNMs. These CNMs were obtained using the self-aggregating nature of carboxylic acid-decorated carbon nanotubes (30 nm diameter and 1-5 $\mu \mathrm{m}$ length) at $\mathrm{pH} 11$. The repetitive dispersion and sonication cycles of the negatively charged nanotubes in DI water facilitated the stable spherical formation. (c) High magnification SEM image of the surface of a CNM, demonstrating densely packed and an entangled network of nanotubes. (d) Cross-sectional image of a CNM after focused ion beam ablation of a portion of the CNM. Consistent with the surface morphology, the nanotubes inside the material are massively packed and well-distributed. The CNM was protected by a Pt layer during the FIB ablation. (e) High magnification of (d); and (f) Histogram showing the distribution of diameters of CNMs. This broad distribution might be narrowed by strictly controlling their spherical growth through various approaches, for example intentional physical/chemical modification of the nanotubes or optimizing experimental conditions. A series of these efforts would obviously enhance the aggregation process of the nanotubes.

Using short $\mathrm{COOH}$-decorated nanotubes $(500 \mathrm{~nm} \sim 1 \mu \mathrm{m})$ resulted in a definite trend toward a decrease in the CNM average diameter $(3.3 \mu \mathrm{m})$ with a variety of different morphologies. If the density of negatively charged species (that is, carboxylic acid groups) is more dependent on nanotube length, this could result in a decrease in ionic strength when short CNTs are used. This may account for the smaller CNM size and less consistent spherical morphology (Figure S2) in Supporting Online Materials (SOM)]. Neutral charged nanotubes and amine-modified MWCNTs (30 nm diameter, $1-5 \mu \mathrm{m}$ length, $>95 \%$ purity; Nanolab Inc, MA) were also used for the formation of microspheres. The lack of a specific chemical affinity resulted in the construction of a thinly spread nanotube film rather than the spherical particulate structures produced at various pHs (Figure S3) in Supporting Online Materials $(\mathrm{SOM})]$. On the other hand, amine $\left(\mathrm{NH}_{2}\right)$ functionalized nanotubes also showed an entangled flat structure instead of forming the spherical assembly (Figure S4) in Supporting Online Materials (SOM)]. The reason is likely attributed to the insufficient charge due to the lack of active amine moieties on the nanotubes. As a derivative of our $\mathrm{COOH}$ functionalized nanotubes, amine-decorated nanotubes were produced through the reaction of the carboxyl $\mathrm{COOH}$ group on the nanotubes with ethylene diamine $\left(\mathrm{H}_{2} \mathrm{~N}-\mathrm{CH}_{2}-\mathrm{CH}_{2}-\mathrm{NH}_{2}\right)$ as a coupling agent that would be responsible for the decrease in the amine concentration. Currently, the mechanism underlying these microspheres is not discovered yet. However, CNM formation is closely related to the surface charge densities around the nanotube. On the other hand, Figure $2 \mathrm{f}$ showed the size distributions of CNMs that were obtained by measuring individual particles by SEM analysis. A statistical histogram revealed a wide range of size distribution, with the average diameter 
of $17.6 \mu \mathrm{m}$. To elucidate the mechanism behind the effect on the particle formation and size, further detailed experimental studies are currently underway in our group. It was recently reported that novel constructs possessing a comparable size to biological structures can readily interact with diverse organic samples - including both chemical and biological moieties [27-30]. Therefore, we investigated whether our novel CNMs would provide a useful and efficient matrix for the simultaneous encapsulation of chemical species within the interior and attached to the exterior of the particles. Indeed, CNMs would make promising candidates for application in broad areas if they were shown to effectively entrap and confine molecules. CNMs composed of nanotubes would be expected to offer significant advantages, especially in biomedical applications: i) relatively long nanotube arrays with an enormous surface area are ideally suited for adsorption of multiple cargo molecules, ii) biocompatible coatings composed of these spheres would offer ideal micro-environment for cell growth and tissue formation, iii) chemical adsorption with biomolecules would produce significantly greater storage stability preventing undesirable degradation or denaturation, and iv) CNMs could be utilized as novel diagnostic tools when combined with imaging agents, such as luminophore or magnetic functionalities. Indeed, the simple and uncomplicated fabrication of CNM using individual nanotubes as molecular building blocks offers finer control over the hybrid compositions and a more standardized production of nanomaterials. As a proof-of-concept experiment in this vein, we functionalized CNMs with representative fluorescent derivatives such as both fluorescein (FITC) and rhodamine (Rho) that were initially conjugated with poly-L-lysine (PLL) and bovine serum albumin (BSA) respectively. The objective was to enhance electrostatic stabilization and simultaneously achieve co-loading of these desired molecules to form large protein complexes. The decorated CNMs were visualized by SEM, conventional bright-field, and fluorescence microscopy (Figures 3 a-f).

SEM images verified the general construction of the CNM-PLL/ FITC complexes but did not reveal whether the proteins were contained within the interior or attached to exterior surfaces of CNMs. However, the complementary evidence provided by fluorescent imaging presented strong fluorescence from FITC-PLL associated with the CNMs (Figure 3c). Like with FITC-PLL loading, the cationic characteristics of RhoBSA complexes resulted in their binding to the negatively charged surface of the nanotubes by electrostatic forces at physiological $\mathrm{pH}$ and confirmed the co-adsorption of negatively charged BSA molecules as shown in Figure 3f. In control experiments, the conjugation with only negatively charged FITC molecules without the PLL led to a very weak signal. As another specific application of such interesting microspheres, we detailed the functionalization of CNMs with positively charged particles, particularly amine-terminated gold nanoparticles $\left(\mathrm{Au}-\mathrm{NH}_{2}\right)$ with $\sim 100 \mathrm{~nm}$ in diameter. SEM images have verified the existence of highly concentrated and uniformly dispersed gold particles at $\mathrm{pH} 11$ (Figure 4). However, the cross-sectional images revealed that most of gold particles were presented on the surface rather than inside of CNT microsphere, assuming that the accumulation of more gold particles inside CNMs might be interrupted by their relatively large size (Figure 4d). And more importantly, novel CNM complexes conjugated with gold nanoparticles exhibited the variation in loading efficiency that can be tuned by altering $\mathrm{pH}$. These results suggested that positively charged moieties derived from guest molecules are definitely responsible for enhancing spherical stability but do not seem to be a critical factor in initiating the microsphere formation.

In parallel experiments, negatively charged silica particles $(150 \mathrm{~nm}$ in a diameter) were used as a control (data not shown). As expected, no detectable quantity of silica particles was adsorbed. Although colloidal stability was not affected, the charge repulsion of similar functional groups inhibited the particle attachment.

\section{Conclusion}

In summary, we offer a convenient method for preparing carbon nanotube microspheres made of only carbon nanotubes. This procedure is in sharp contrast to conventional methods which focus on breaking bundles into individual nanotubes. Rather we exploit the spontaneous self-aggregation characteristics of nanotubes to merge them into colloidal structures by manipulation of the $\mathrm{pH}$. Such thermodynamically and kinetically efficient fabrication offers new promises in discovery and design of CNM hybrids by simply engineering the structural geometry or chemical integrity. In addition,
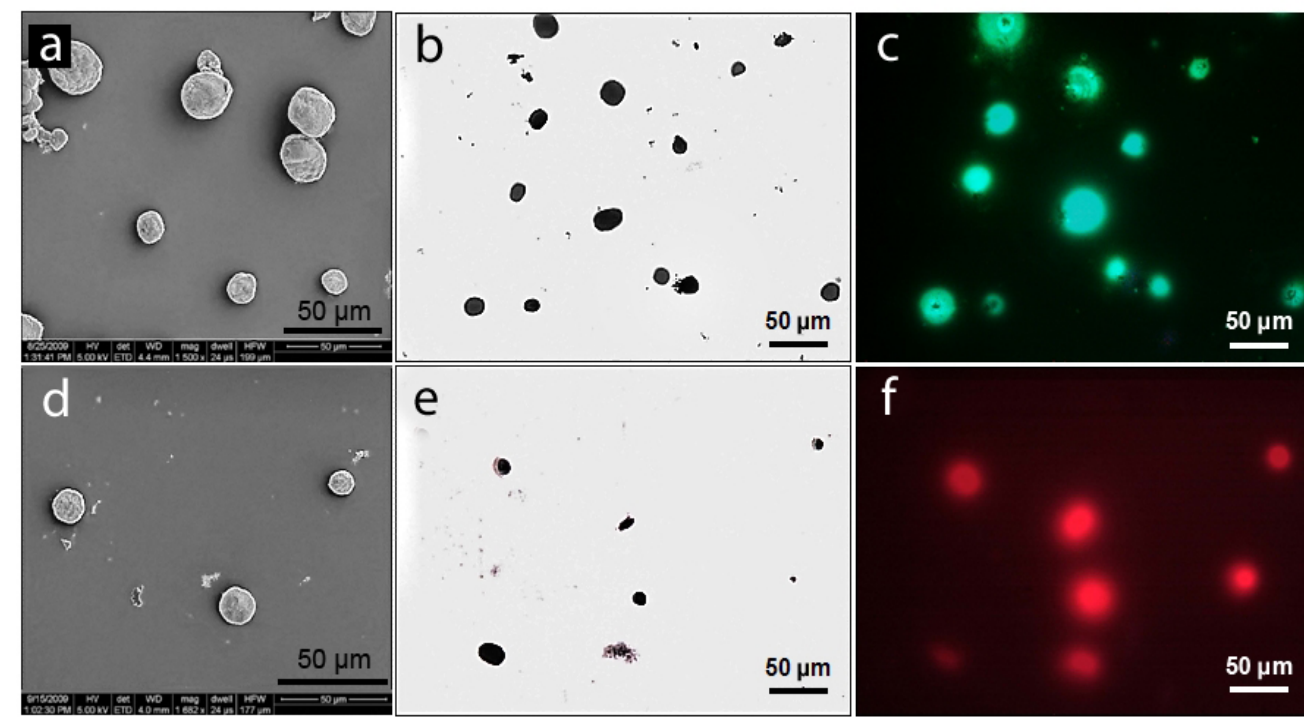

e
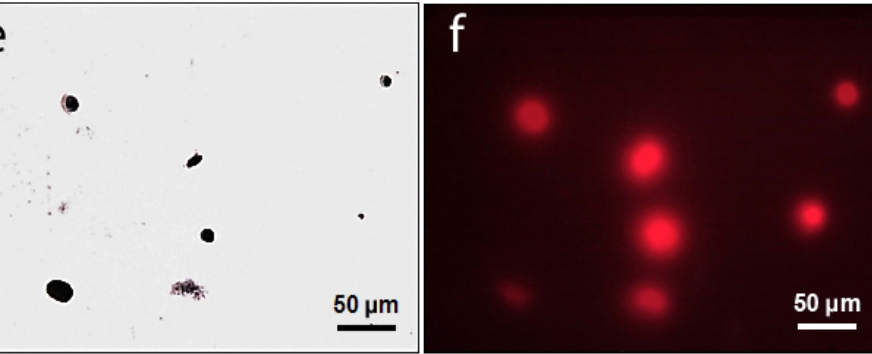

Figure 3. CNM-protein complexes prepared by incubation with (a c) FITC / poly- $L$-lysine (PLL) and (d f) rhodamine (rho) / bovine serum albumin (BSA). Images (a) and (d) were by SEM; while images (b) and (e) were collected by conventional optical microscopy. Images (c) and (f) were obtained by fluorescence microscopy. The strong fluorescent intensity confirmed sufficient conjugation of PLL and BSA onto the CNMs. 


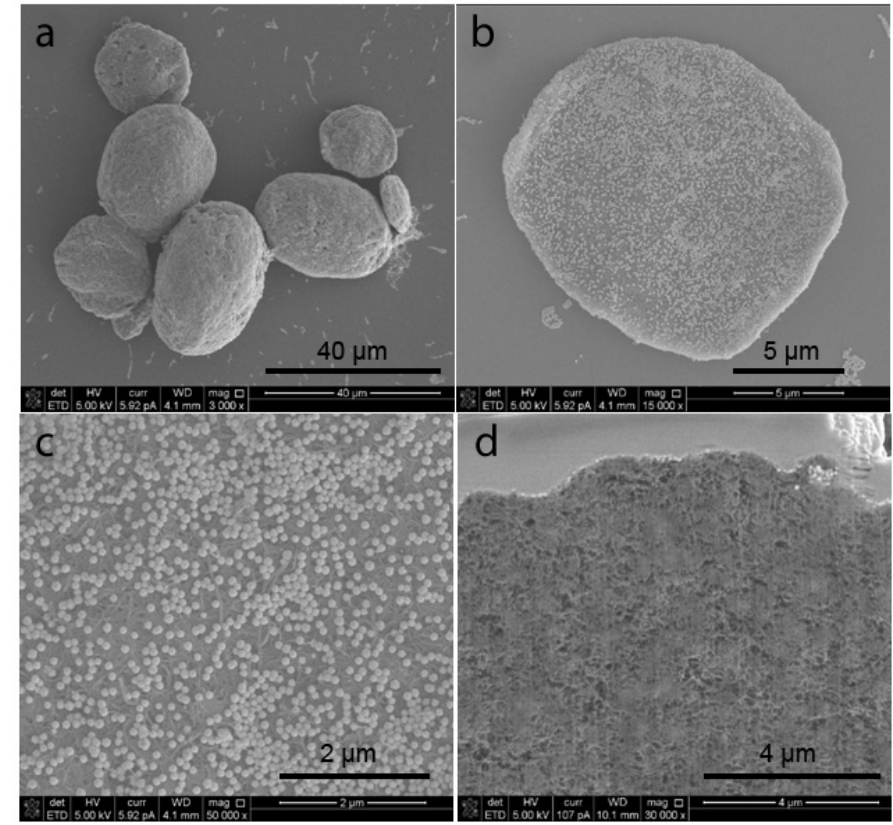

Figure 4. (a) (b) SEM images of CNMs conjugated with $100 \mathrm{~nm} \mathrm{NH2-decorated} \mathrm{gold}$ nanoparticles. (c) High magnification SEM image of the external surface of a CNM, demonstrating the presence of gold nanoparticles. (d) High magnification cross-sectional images of a CNM after focused ion beam ablation of a portion of the CNM

the fact that our approach is accessible to innumerable guest molecules extends this new variation of CNM complex to diverse endeavors, especially chemistry, biotechnology, electronics, and of great importance - therapeutic approacheS.

\section{Acknowledgement}

This work was supported by a National Cancer Center grant from the Republic of Korea (1611170-3) and a grant of the Korea Health technology R\&D Project through the Korea Health Industry Development Institute(KHIDI), funded by the Ministry of Health \& Welfare, Republic of Korea (Grant Number: HI17C0828).

\section{Compliance with Ethical Standards}

\section{Conflict of Interest}

The authors declare that they have no conflict of interest.

\section{Supporting Information Available}

SEM images of CNMs and nanotube surface produced from pristine multiwalled carbon nanotubes and the nanotube surface produced from amine.

\section{References}

1. Bethune DS, Klang CH, Vries MS, Gorman RS, Beyers JVR (1996) Cobalt-catalysed growth of carbon nanotubes with single-atomic-layer walls. Nature 363: 605-607.

2. Iijima S (1991) Helical microtubules of graphitic carbon. Nature 354: 56-58.

3. Zhang Y, Ichihashi T, Landree E, Nihey F, Iijima S (1999) Heterostructures of SingleWalled Carbon Nanotubes and Carbide Nanorods. Science 285: 1719-1722.

4. Avouris P, Chen Z, Perebeinos V (2007) Carbon-based electronics. Nature nanotech 2: 605-615.

5. Besteman K, Lee J, Wiertz FGM, Heering HA, Dekker C (2003) Enzyme-coated carbon nanotubes as single-molecule biosensors. Nano Lett 3: 727-730.

6. Gao H, Kong Y (2004) Simulations of DNA-Nanotube interactions. Annu Rev Mater Sci 34: $123-150$
7. Ramesh S, Ericson LM, Davis VA, Saini R K, Kittrell C, et al. (2004) Dissolution of Pristine Single Walled Carbon Nanotubes in Superacids by Direct Protonation. $J$ Phys Chem B 108: 8794-8798.

8. Sun Z, Nicolosi V, Rickard D, Bergin SD, Aherne D, et al. (2008) Quantitative evaluation of surfactant-stabilized single-walled carbon nanotubes: Dispersion quality and its correlation with Zeta potential. J Phys Chem C 112: 10692-10699.

9. Tummala NR, Striolo A (2009) SDS surfactants on carbon nanotubes: aggregate morphology. ACS Nano 3: 595-602.

10. Ericson LM, Fan H, Peng H, Davis VA, Zhou W, et al. (2004) Macroscopic, Neat, Single-Walled Carbon Nanotube Fibers. Science 305: 1447-1450

11. Futaba DN, Hata K, Yamada T, Hiraoka T, Hayamizu Y, et al. (2006) Shape-engineerable and highly densely packed single-walled carbon nanotubes and their application as super-capacitor electrodes. Nature mater 5: 987-994.

12. Richard C, Balavoine F, Schultz P, Ebbesen TW, Mioskowski C (2003) Supramolecular Self-Assembly of Lipid Derivatives on Carbon Nanotubes. Science 300: 775-778.

13. Sano M, Kamino A, Okamura J, Shinkai S (2001) Ring Closure of Carbon Nanotubes. Science 293: 1299-1301.

14. Sano M, Kamino A, Okamura J, Shinkai S (2002) Noncovalent Self-Assembly of Carbon Nanotubes for Construction of "Cages". Nano Lett 2: 531-533.

15. Vigolo B, Pénicaud A, Coulon C, Sauder C, Pailler R, et al. (2000) Macroscopic Fibers and Ribbons of Oriented Carbon Nanotubes. Science 290: 1331-1334.

16. Correa-Duarte MA, Kosioreck A, Kandulski W, Giersig M, Salgueirini-Maceira V (2006) Nanoengineered polymetric thin films by sintering CNT-coated polystyrene spheres. Small 2: 220-224.

17. Jan E, Kotov NA (2007) Successful Differentiation of Mouse Neural Stem Cells on Layer-by-Layer Assembled Single-Walled Carbon Nanotube Composite. Nano Lett 7 : 1123-1128.

18. Rouse JH, Lillehei PT (2003) Electrostatic Assembly of Polymer/Single Walled Carbon Nanotube Multilayer Films. Nano Lett 3: 59-62.

19. Cadek M, Coleman JN, Ryan KP, Nicolosi V, Bister G, et al. (2004) Reinforcement of Polymers with Carbon Nanotubes: The Role of Nanotube Surface Area. Nano Lett 4: 353-356.

20. Tang W, Santare MH, Advani SG (2003) Melt processing and mechanical property characterization of multi-walled carbon nanotube/high density polyethylene (MWNT/ HDPE) composite films. Carbon 41: 2779-2785.

21. Correa-Duarte MA, Kosiorek A, Kandulski W, Giersig M, Liz-Marzan LM (2005) Layer-by-layer assembly of multiwall carbon nanotubes on spherical colloids. Chem Mater 17: 3268-3272.

22. Ji L, Ma J, Wei W, Ji L, Wang X, et al. (2006) Porous hollow carbon nanotube composite cages. Chem Commun 1206-1208.

23. Panhuis M, Paunov VN (2005) Assembling carbon nanotubesomes using an emulsioninversion technique. Chem Commun 1726-1728.

24. Sano M, Kamino A, Shinkai S (2001) Construction of carbon nanotubes "stars" with dendrimers. Angew. Chem Int Ed 40: 4661-4663.

25. Yang S, Song H, Yi H, Liu W, Zhang H, et al. (2009) Carbon nanotube capsules encapsulating $\mathrm{SnO} 2$ nanoparticles as an anode material for lithium ion batteries. Electrochimica Acta 55: 521-527.

26. Zuberi M, Sherman D, Cho Y (2011) Carbon Nanotube Microspheres Produced by Surfactant-Mediated Aggregation. J Phys Chem C 115: 3881-3887.

27. Khlobystov AN, Britz DA, Briggs GAD (2005) Molecules in carbon nanotubes. Acc Chem Res 38: 901-909.

28. Liu Z, Cai W, He L, Nakayama N, Chen K, et al. (2007) In vivo biodistribution and highly efficient tumor targeting of carbon nanotubes in mice. Nat Nanotechnol 2: 47 52. [Crossref]

29. Liu Z, Sun X, Nakayama-Ratchford N, Dai H (2007) Supramolecular chemistry on water-soluble carbon nanotubes for drug loading and delivery. ACS Nano 1: 50-56.

30. Prato M, Kostarelos K, Bianco A (2008) Functionalized carbon nanotubes in drug design and discovery. Acc Chem Res 41: 60-68.

Copyright: (C2018 Choi M. This is an open-access article distributed under the terms of the Creative Commons Attribution License, which permits unrestricted use, distribution, and reproduction in any medium, provided the original author and source are credited. 